\title{
ON SOME PRODUCTS OF COMMUTATORS IN AN ASSOCIATIVE RING
}

\author{
GALINA DERYABINA AND ALEXEI KRASILNIKOV
}

\begin{abstract}
Let $A$ be a unital associative ring and let $T^{(k)}$ be the two-sided ideal of $A$ generated by all commutators $\left[a_{1}, a_{2}, \ldots, a_{k}\right]\left(a_{i} \in A\right)$ where $\left[a_{1}, a_{2}\right]=a_{1} a_{2}-a_{2} a_{1},\left[a_{1}, \ldots, a_{k-1}, a_{k}\right]=\left[\left[a_{1}, \ldots, a_{k-1}\right], a_{k}\right]$ $(k>2)$. It has been known that, if either $m$ or $n$ is odd then

$$
6\left[a_{1}, a_{2}, \ldots, a_{m}\right]\left[b_{1}, b_{2}, \ldots, b_{n}\right] \in T^{(m+n-1)}
$$
\end{abstract}

for all $a_{i}, b_{j} \in A$. This was proved by Sharma and Srivastava in 1990 and independently rediscovered later (with different proofs) by various authors. The aim of our note is to give a simple proof of the following result: if at least one of the integers $m, n$ is odd then, for all $a_{i}, b_{j} \in A$,

$$
3\left[a_{1}, a_{2}, \ldots, a_{m}\right]\left[b_{1}, b_{2}, \ldots, b_{n}\right] \in T^{(m+n-1)} .
$$

Since it has been known that, in general,

$$
\left[a_{1}, a_{2}, a_{3}\right]\left[b_{1}, b_{2}\right] \notin T^{(4)},
$$

our result cannot be improved further for all $m, n$ such that at least one of them is odd.

2010 AMS MSC Classification: 16R10, 16R40

Keywords: polynomial identity, commutator, Lie nilpotent associative ring

\section{INTRODUCTION}

Let $A$ be a unital associative ring. Define a left-normed commutator $\left[a_{1}, \ldots, a_{k-1}, a_{k}\right]\left(k>1, a_{i} \in A\right.$ for all $i$ ) recursively as follows: $\left[a_{1}, a_{2}\right]=a_{1} a_{2}-a_{2} a_{1},\left[a_{1}, \ldots, a_{k-1}, a_{k}\right]=\left[\left[a_{1}, \ldots, a_{k-1}\right], a_{k}\right](k>2)$. Let $T^{(k)}=T^{(k)}(A)$ be the two-sided ideal of $A$ generated by all commutators $\left[a_{1}, a_{2}, \ldots, a_{k}\right]\left(a_{i} \in A\right)$.

For each unital associative $\operatorname{ring} A$, all $m, n \in \mathbb{Z}, m, n>1$ and all $a_{i}, b_{j} \in A$, we have

$$
\left[a_{1}, \ldots, a_{m}\right]\left[b_{1}, \ldots, b_{n}\right] \in T^{(m+n-2)} .
$$

This result was proved by Latyshev [15, Lemma 1] in 1965 in an article published in Russian and independently rediscovered by Gupta and Levin [13, Theorem 3.2] in 1983.

If $m=3$ then, by (1), we have $\left[a_{1}, a_{2}, a_{3}\right]\left[b_{1}, b_{2}, \ldots, b_{n}\right] \in T^{(n+1)}$. Note that $T^{(n+1)} \supseteq T^{(n+2)}$. In 1985 Levin and Sehgal [16, Lemma 2(a)] proved that, for all $n>1$ and all $a_{i}, b_{j} \in A$,

$$
3\left[a_{1}, a_{2}, a_{3}\right]\left[b_{1}, b_{2}, \ldots, b_{n}\right] \in T^{(n+2)} .
$$

Earlier, in 1978, a similar result was proved by Volichenko [19, Lemma 1] in a preprint written in Russian. More recently, some particular cases of this result were independently rediscovered, with different proofs, in [10, Theorem 3.4] and [11, Lemma 1].

In 1990 Sharma and Srivastava [18, Theorem 2.8] proved that if $m, n \in \mathbb{Z}, m, n>0$ and at least one of $m, n$ is odd then, for all $a_{i}, b_{j} \in A$,

$$
6\left[a_{1}, \ldots, a_{m}\right]\left[b_{1}, \ldots, b_{n}\right] \in T^{(m+n-1)} .
$$

Recently this result was independently rediscovered, with different proofs, in [2, Corollary 1.4] and [12, Theorem 1].

Note that if $m=2 m^{\prime}, n=2 n^{\prime}$ both are even then the result similar to (2) does not hold: there exit associative algebras $A=A(m, n)$ over a field $F$ of characteristic 0 such that, for some $a_{i}, b_{j} \in A$,

$$
\left[a_{1}, \ldots, a_{2 m^{\prime}}\right]\left[b_{1}, \ldots, b_{2 n^{\prime}}\right] \notin T^{\left(2 m^{\prime}+2 n^{\prime}-1\right)}
$$


(see [8, Theorem 1.4] or [12, Lemma 6]) so

$$
\ell\left[a_{1}, \ldots, a_{2 m^{\prime}}\right]\left[b_{1}, \ldots, b_{2 n^{\prime}}\right] \notin T^{\left(2 m^{\prime}+2 n^{\prime}-1\right)}
$$

for all $\ell \in \mathbb{Z}, \ell \neq 0$.

The aim of the present note is to give a simple proof of the following theorem that improves the result (2) by Sharma and Srivastava.

Theorem 1.1. Let $A$ be a unital associative ring. Let $m, n \in \mathbb{Z}, m, n>0$. Suppose that at least one of the integers $m, n$ is odd. Then, for all $a_{i}, b_{j} \in A$,

$$
3\left[a_{1}, \ldots, a_{m}\right]\left[b_{1}, \ldots, b_{n}\right] \in T^{(m+n-1)} .
$$

Note that, for some $A$ and some $a_{i}, b_{j} \in A$,

$$
\left[a_{1}, a_{2}, a_{3}\right]\left[b_{1}, b_{2}\right] \notin T^{(4)}
$$

(see [14, Theorem 1.1]) so for $m=3, n=2$ Theorem 1.1 cannot be improved. On the other hand, if $m=n=3$ then, for each associative ring $A$ and all $a_{i}, b_{j} \in A$,

$$
\left[a_{1}, a_{2}, a_{3}\right]\left[b_{1}, b_{2}, b_{3}\right] \in T^{(5)}
$$

(see [5, Lemma 2.1]). There is some evidence that suggests that the case $m=n=3$ is exceptional and in all other cases Theorem 1.1 cannot be improved further.

Conjecture 1.2. Let $m, n \in \mathbb{Z}, m, n>1$ and either $m$ or $n$ is odd. If $(m, n) \neq(3,3)$ then there is a unital associative ring $A$ and $a_{i}, b_{j} \in A$ such that

$$
\left[a_{1}, \ldots, a_{m}\right]\left[b_{1}, \ldots, b_{n}\right] \notin T^{(m+n-1)}
$$

It is easy to check that to prove (or disprove) Conjecture 1.2 one can assume that $A=\mathbb{Z}\langle X, Y\rangle$ is the free unital associative ring on a free generating set $X \cup Y$ where $X=\left\{x_{1}, x_{2}, \ldots\right\}, Y=\left\{y_{1}, y_{2}, \ldots\right\}$ and $a_{i}=x_{i}, b_{j}=y_{j}$ for all $i, j$.

The following assertion follows immediately from Theorem 1.1] (see [9, Prop. 1.3 and 1.4] for more details).

Corollary 1.3. Let $A$ be a unital associative ring and let $n_{1}, \ldots, n_{k} \in \mathbb{Z}, n_{i}>0$ for all $i$. Suppose that $\ell$ of the integers $n_{1}, \ldots, n_{k}$ are odd. Let

$$
q= \begin{cases}\ell & \text { if } \ell<k, \text { that is, if at least one } n_{i} \text { is even; } \\ \ell-1 & \text { if } \ell=k, \text { that is, if all } n_{i} \text { are odd }\end{cases}
$$

and let $N_{k, q}=n_{1}+\cdots+n_{k}-2(k-1)+q$. Then, for all $a_{i j} \in A$,

$$
3^{q}\left[a_{11}, \ldots, a_{1 n_{1}}\right] \ldots\left[a_{k 1}, \ldots, a_{k n_{k}}\right] \in T^{\left(N_{k, q}\right)} .
$$

Note that, in general,

$$
r\left[a_{11}, \ldots, a_{1 n_{1}}\right] \ldots\left[a_{k 1}, \ldots, a_{k n_{k}}\right] \notin T^{\left(N_{k, q}+1\right)}
$$

for some unital associative ring $A$, some $a_{i j} \in A$ and all $r \in \mathbb{Z}, r \neq 0$. This was proved by Dangovski [6. Prop. 2.2] if $\ell=k$ and by the authors of the present article [9, Theorem 1.7] if $\ell<k$.

Remarks. 1. Theorem 1.1 and Conjecture 1.2 are closely connected to the description of the additive group of the ring $\mathbb{Z}\langle X\rangle / T^{(k)}$ where $\mathbb{Z}\langle X\rangle$ is the free unital associative ring with a free generating set $X=\left\{x_{1}, x_{2}, \ldots\right\}$.

It is clear that the additive group of the ring $\mathbb{Z}\langle X\rangle / T^{(2)}$ is free abelian. It was shown in 3 that the additive group of $\mathbb{Z}\langle X\rangle / T^{(3)}$ is also free abelian. On the other hand, the additive group of the ring $\mathbb{Z}\langle X\rangle / T^{(4)}$ is a direct sum $G \oplus H$ of a free abelian group $G$ and an elementary abelian 3-group $H$ (see [7, 14]). Computational data by Cordwell, Fei and Zhou presented in [4, Appendix A] suggest that for $k \geq 6$ the additive group of the ring $\mathbb{Z}\langle X\rangle / T^{(k)}$ is also a direct sum of a free abelian group $G$ and a non-trivial elementary abelian 3-group $H$ while for $k=5$ this group is free abelian. However, it is 
still an open problem whether the torsion subgroup $H$ of the additive group of $\mathbb{Z}\langle X\rangle / T^{(k)}$ is indeed a non-trivial (elementary) abelian 3-group if $k>5$ and $H=\{0\}$ if $k=5$.

If Conjecture 1.2 holds then the elements $\left[a_{1}, \ldots, a_{m}\right]\left[b_{1}, \ldots, b_{n}\right]+T^{(m+n-1)}$ are non-trivial elements of $H \subset \mathbb{Z}\langle X\rangle / T^{(m+n-1)}$ whose order, by Theorem 1.1, is equal to 3 (if $(m, n) \neq(3,3)$ ). If $m=3$, $n=2$ then such products of commutators generate $H$ as a two-sided ideal in $\mathbb{Z}\langle X\rangle / T^{(4)}$ (see [7, 14]). One might expect a similar situation if $k>5$.

2. The proof of (2) given in [2] can be modified to prove Theorem 1.1 (see [1, Remark 3.9] for explanation). This modification uses computer calculations in a free associative ring. Our proof of Theorem 1.1 does not require computer calculations and is much simpler then that modification of the proof given in [2].

3. Theorem 1.1 and its corollary remain valid for a non-unital associative ring $A$; one can easily deduce this from the corresponding results for unital rings. We state and prove our results for a unital associative ring $A$ in order to simplify notation in the proof.

\section{Proof of Theorem 1.1}

It is straightforward to check that

$$
\left[a_{1} a_{2}, b\right]=a_{1}\left[a_{2}, b\right]+\left[a_{1}, b\right] a_{2}
$$

so the map $D_{b}: A \rightarrow A$ such that $D_{b}(a)=[a, b](a \in A)$ is a derivation of the $\operatorname{ring} A$. It follows that

$$
\begin{gathered}
{\left[a_{1} a_{2}, b_{1}, b_{2}\right]=a_{1}\left[a_{2}, b_{1}, b_{2}\right]+\left[a_{1}, b_{1}\right]\left[a_{2}, b_{2}\right]+\left[a_{1}, b_{2}\right]\left[a_{2}, b_{1}\right]+\left[a_{1}, b_{1}, b_{2}\right] a_{2}} \\
{\left[a_{1} a_{2}, b_{1}, b_{2}, b_{3}\right]=} \\
a_{1}\left[a_{2}, b_{1}, b_{2}, b_{3}\right]+\left[a_{1}, b_{1}\right]\left[a_{2}, b_{2}, b_{3}\right]+\left[a_{1}, b_{2}\right]\left[a_{2}, b_{1}, b_{3}\right] \\
+\left[a_{1}, b_{3}\right]\left[a_{2}, b_{1}, b_{2}\right]+\left[a_{1}, b_{1}, b_{2}\right]\left[a_{2}, b_{3}\right]+\left[a_{1}, b_{1}, b_{3}\right]\left[a_{2}, b_{2}\right] \\
+\left[a_{1}, b_{2}, b_{3}\right]\left[a_{2}, b_{1}\right]+\left[a_{1}, b_{1}, b_{2}, b_{3}\right] a_{2}
\end{gathered}
$$

for all $a_{i}, b_{j} \in A$.

The following lemma is a modification of well-known results (see, for instance, [14, Lemma 2.1], 15, Lemma 2 (3)], [17, Lemma 8.2]). We prove it here in order to have the article self-contained.

Lemma 2.1. Let $A$ be a unital associative ring. Then, for all $k>1$ and all $g_{i}, f_{j} \in A$, we have

$$
\begin{aligned}
& {\left[g_{1}, \ldots, g_{k-1}, f_{1}\right]\left[f_{2}, f_{3}, f_{4}\right]+\left[g_{1}, \ldots, g_{k-1}, f_{2}\right]\left[f_{1}, f_{3}, f_{4}\right] \in T^{(k+2)},} \\
& {\left[g_{1}, \ldots, g_{k-1}, f_{1}\right]\left[f_{2}, f_{3}, f_{4}\right]+\left[g_{1}, \ldots, g_{k-1}, f_{4}\right]\left[f_{2}, f_{3}, f_{1}\right] \in T^{(k+2)} .}
\end{aligned}
$$

Proof. Let $c_{1}=\left[g_{1}, \ldots, g_{k-1}, g_{k}\right]$. It is clear that $\left[c_{1},\left(h_{1} h_{2}\right), h_{3}\right] \in T^{(k+2)}$ for all $g_{i}, h_{j} \in A$. We have $\left[c_{1},\left(h_{1} h_{2}\right), h_{3}\right]=-\left[h_{1} h_{2}, c_{1}, h_{3}\right]$ and, by (4),

$$
\left[h_{1} h_{2}, c_{1}, h_{3}\right]=h_{1}\left[h_{2}, c_{1}, h_{3}\right]+\left[h_{1}, c_{1}\right]\left[h_{2}, h_{3}\right]+\left[h_{1}, h_{3}\right]\left[h_{2}, c_{1}\right]+\left[h_{1}, c_{1}, h_{3}\right] h_{2} \in T^{(k+2)} .
$$

It is clear that $h_{1}\left[h_{2}, c_{1}, h_{3}\right]=-h_{1}\left[c_{1}, h_{2}, h_{3}\right] \in T^{(k+2)}$; similarly, $\left[h_{1}, c_{1}, h_{3}\right] h_{2} \in T^{(k+2)}$ so

$$
\left[h_{1}, c_{1}\right]\left[h_{2}, h_{3}\right]+\left[h_{1}, h_{3}\right]\left[h_{2}, c_{1}\right] \in T^{(k+2)} \text {. }
$$

It follows that

$$
\left[c_{1}, h_{1}\right]\left[h_{2}, h_{3}\right]+\left[c_{1}, h_{2}\right]\left[h_{1}, h_{3}\right]-\left[\left[c_{1}, h_{2}\right],\left[h_{1}, h_{3}\right]\right]=-\left(\left[h_{1}, c_{1}\right]\left[h_{2}, h_{3}\right]+\left[h_{1}, h_{3}\right]\left[h_{2}, c_{1}\right]\right) \in T^{(k+2)} .
$$

Since

we have

$$
\left[\left[c_{1}, h_{2}\right],\left[h_{1}, h_{3}\right]\right]=\left[c_{1}, h_{2}, h_{1}, h_{3}\right]-\left[c_{1}, h_{2}, h_{3}, h_{1}\right] \in T^{(k+3)} \subseteq T^{(k+2)},
$$

$$
\left[c_{1}, h_{1}\right]\left[h_{2}, h_{3}\right]+\left[c_{1}, h_{2}\right]\left[h_{1}, h_{3}\right] \in T^{(k+2)} .
$$


Now we check that (7) holds. Let $c_{2}=\left[g_{1}, \ldots, g_{k-1}\right]$. It is clear that $\left[c_{2},\left(f_{1} f_{2}\right), f_{3}, f_{4}\right] \in T^{(k+2)}$ for all $g_{i}, f_{j} \in A$. We have $\left[c_{2},\left(f_{1} f_{2}\right), f_{3}, f_{4}\right]=-\left[f_{1} f_{2}, c_{2}, f_{3}, f_{4}\right]$ and, by (5),

$$
\begin{aligned}
{\left[f_{1} f_{2}, c_{2}, f_{3}, f_{4}\right]=f_{1}[} & \left.f_{2}, c_{2}, f_{3}, f_{4}\right]+\left[f_{1}, c_{2}\right]\left[f_{2}, f_{3}, f_{4}\right] \\
& +\left[f_{1}, f_{3}\right]\left[f_{2}, c_{2}, f_{4}\right] \\
& +\left[f_{1}, f_{4}\right]\left[f_{2}, c_{2}, f_{3}\right]+\left[f_{1}, c_{2}, f_{3}\right]\left[f_{2}, f_{4}\right]+\left[f_{1}, c_{2}, f_{4}\right]\left[f_{2}, f_{3}\right] \\
& +\left[f_{1}, f_{3}, f_{4}\right]\left[f_{2}, c_{2}\right]+\left[f_{1}, c_{2}, f_{3}, f_{4}\right] f_{4} \in T^{(k+2)} .
\end{aligned}
$$

It is clear that $f_{1}\left[f_{2}, c_{2}, f_{3}, f_{4}\right]=-f_{1}\left[c_{2}, f_{2}, f_{3}, f_{4}\right] \in T^{(k+2)}$ and similarly $\left[f_{1}, c_{2}, f_{3}, f_{4}\right] f_{4} \in T^{(k+2)}$. Further, by (요),

$$
\begin{aligned}
{\left[f_{1}, f_{3}\right]\left[f_{2}, c_{2}, f_{4}\right]+\left[f_{1}, f_{4}\right]\left[f_{2}, c_{2}, f_{3}\right]=\left(\left[c_{2}, f_{2}, f_{4}\right]\left[f_{3}, f_{1}\right]+\left[c_{2}, f_{2}, f_{3}\right]\left[f_{4}, f_{1}\right]\right) } & \\
- & -\left[\left[c_{2}, f_{2}, f_{4}\right],\left[f_{3}, f_{1}\right]\right]-\left[\left[c_{2}, f_{2}, f_{3}\right],\left[f_{4}, f_{1}\right]\right] \in T^{(k+2)}
\end{aligned}
$$

and similarly $\left[f_{1}, c_{2}, f_{3}\right]\left[f_{2}, f_{4}\right]+\left[f_{1}, c_{2}, f_{4}\right]\left[f_{2}, f_{3}\right]=\left[c_{2}, f_{1}, f_{3}\right]\left[f_{4}, f_{2}\right]+\left[c_{2}, f_{1}, f_{4}\right]\left[f_{3}, f_{2}\right] \in T^{(k+2)}$. It follows that

$$
\left[f_{1}, c_{2}\right]\left[f_{2}, f_{3}, f_{4}\right]+\left[f_{1}, f_{3}, f_{4}\right]\left[f_{2}, c_{2}\right] \in T^{(k+2)}
$$

so

$$
\begin{aligned}
{\left[c_{2}, f_{1}\right]\left[f_{2}, f_{3}, f_{4}\right]+\left[c_{2}, f_{2}\right]\left[f_{1}, f_{3}, f_{4}\right]-\left[\left[c_{2}, f_{2}\right],\right.} & {\left.\left[f_{1}, f_{3}, f_{4}\right]\right] } \\
& =-\left(\left[f_{1}, c_{2}\right]\left[f_{2}, f_{3}, f_{4}\right]+\left[f_{1}, f_{3}, f_{4}\right]\left[f_{2}, c_{2}\right]\right) \in T^{(k+2)} .
\end{aligned}
$$

Since $\left[\left[c_{2}, f_{2}\right],\left[f_{1}, f_{3}, f_{4}\right]\right] \in T^{(k+3)} \subseteq T^{(k+2)}$, we have

$$
\left[c_{2}, f_{1}\right]\left[f_{2}, f_{3}, f_{4}\right]+\left[c_{2}, f_{2}\right]\left[f_{1}, f_{3}, f_{4}\right] \in T^{(k+2)}
$$

so (17) holds.

It remains to check that ([6) holds. Recall that $c_{2}=\left[g_{1}, \ldots, g_{k-1}\right]$. By the Jacobi identity,

$$
\left[f_{2}, f_{3}, f_{4}\right]=\left[f_{4}, f_{3}, f_{2}\right]-\left[f_{4}, f_{2}, f_{3}\right], \quad\left[f_{2}, f_{3}, f_{1}\right]=\left[f_{1}, f_{3}, f_{2}\right]-\left[f_{1}, f_{2}, f_{3}\right]
$$

so

$$
\begin{aligned}
{\left[c_{2}, f_{1}\right]\left[f_{2}, f_{3}, f_{4}\right] } & +\left[c_{2}, f_{4}\right]\left[f_{2}, f_{3}, f_{1}\right] \\
& =\left(\left[c_{2}, f_{1}\right]\left[f_{4}, f_{3}, f_{2}\right]+\left[c_{2}, f_{4}\right]\left[f_{1}, f_{3}, f_{2}\right]\right)-\left(\left[c_{2}, f_{1}\right]\left[f_{4}, f_{2}, f_{3}\right]+\left[c_{2}, f_{4}\right]\left[f_{1}, f_{2}, f_{3}\right]\right) .
\end{aligned}
$$

By (17), we have $\left[c_{2}, f_{1}\right]\left[f_{2}, f_{3}, f_{4}\right]+\left[c_{2}, f_{4}\right]\left[f_{2}, f_{3}, f_{1}\right] \in T^{(k+2)}$, that is, (6) holds.

This completes the proof of Lemma 2.1.

Corollary 2.2. Let $A$ be a unital associative ring. Then, for all $k>1$ and all $g_{i}, f_{j} \in A$ and for each permutation $\sigma$ on the set $\{1,2,3,4\}$, we have

$$
\left[g_{1}, \ldots, g_{k-1}, f_{\sigma(1)}\right]\left[f_{\sigma(2)}, f_{\sigma(3)}, f_{\sigma(4)}\right] \equiv(-1)^{\sigma}\left[g_{1}, \ldots, g_{k-1}, f_{1}\right]\left[f_{2}, f_{3}, f_{4}\right] \quad\left(\bmod T^{(k+2)}\right) .
$$

Proof. It is clear that (9) is true if $\sigma=(23)$ is the transposition that permutes 2 and 3. By Lemma 2.1. (9) holds if $\sigma=(12)$ and $\sigma=(14)$. Hence, (9) is true for all permutations $\sigma$ that are products of the transpositions (12), (23) and (14). However, it is easy to check that these 3 transpositions generate the group $S_{4}$ of all permutations on the set $\{1,2,3,4\}$. The result follows.

The following corollary has been proved by Levin and Sehgal [16, Lemma 2(a)]. Our proof is different from one given in [16; it shows that the coefficient 3 in (10) appears because of the Jacobi identity.

Corollary 2.3 (see [16]). Let $A$ be a unital associative ring. Then, for all $a_{i}, b_{j} \in A$,

$$
3\left[a_{1}, \ldots, a_{k}\right]\left[b_{1}, b_{2}, b_{3}\right] \in T^{(k+2)} .
$$


Proof. By the Jacobi identity,

$$
\left[a_{1}, \ldots, a_{k}\right]\left(\left[b_{1}, b_{2}, b_{3}\right]+\left[b_{2}, b_{3}, b_{1}\right]+\left[b_{3}, b_{1}, b_{2}\right]\right)=0 .
$$

On the other hand, by Corollary 2.2 ,

$$
\left.\left[a_{1}, \ldots, a_{k}\right]\left[b, b_{2}, b_{3}\right] \equiv\left[a_{1}, \ldots, a_{k}\right]\left[b_{2}, b_{3}, b_{1}\right] \quad\left(\bmod T^{(k+2)}\right) \equiv\left[a_{1}, \ldots, a_{k}\right]\left[b_{3}, b_{1}, b_{2}\right]\right) \quad\left(\bmod T^{(k+2)}\right) .
$$

It follows that

$$
3\left[a_{1}, \ldots, a_{k}\right]\left[b_{1}, b_{2}, b_{3}\right] \equiv 0 \quad\left(\bmod T^{(k+2)}\right),
$$

as required.

The following lemma is a modification of [12, Lemma 2]. Note that the proof given in [12] allows to prove only the inclusion $6\left[T^{(k)}, A, A\right] \subseteq T^{(k+2)}$.

Lemma 2.4 (cf. [12]). Let $A$ be a unital associative ring. Then, for each $k \geq 1$, we have

$$
3\left[T^{(k)}, A, A\right] \subseteq T^{(k+2)} .
$$

Remark. In general, for $\ell \in \mathbb{Z}, \ell \neq 0$,

$$
\ell\left[T^{(k)}, A\right] \nsubseteq T^{(k+1)}
$$

More precisely, if $k=2 k^{\prime}$ is even then, in general,

$$
\ell\left[T^{\left(2 k^{\prime}\right)}, A\right] \nsubseteq T^{\left(2 k^{\prime}+1\right)}
$$

for any $\ell \in \mathbb{Z}, \ell \neq 0$. This can be easily deduced from (3).

On the other hand, if $k=2 k^{\prime}+1$ is odd then

$$
3\left[T^{\left(2 k^{\prime}+1\right)}, A\right] \subseteq T^{\left(2 k^{\prime}+2\right)} ;
$$

however, to prove this one has to use Theorem 1.1

Proof of Lemma 2.4. By definition, $T^{(k)}$ is the two-sided ideal of $A$ generated by all commutators $\left[a_{1}, a_{2}, \ldots, a_{k}\right]\left(a_{i} \in A\right)$. However, one can easily check that $T^{(k)}$ is generated by the commutators $\left[a_{1}, a_{2}, \ldots, a_{k}\right]\left(a_{i} \in A\right)$ as a right ideal in $A$ as well. Hence, to prove the lemma it suffices to prove that

$$
3[c u, v, w] \in T^{(k+2)}
$$

where $u, v, w \in A, c=\left[a_{1}, a_{2}, \ldots, a_{k}\right]\left(a_{i} \in A\right)$. We have

$$
[c u, v, w]=c[u, v, w]+[c, v][u, w]+[c, w][u, v]+[c, v, w] u .
$$

By Corollary 2.3, we have

$$
3 c[u, v, w] \in T^{(k+2)} .
$$

It is clear that

$$
[c, v, w] u \in T^{(k+2)} .
$$

Further,

$$
[c, v][u, w]+[c, w][u, v]=[v, c][w, u]+[v, u][w, c]+[[c, w],[u, v]]
$$

where $[[c, w],[u, v]] \in T^{(k+3)} \subseteq T^{(k+2)}$ and, by (4),

$$
\begin{aligned}
{[v, c][w, u]+[v, u][w, c]=[v w, c, u]-v[w, c, u]-[v, c, u] w } & \\
& =-[c, v w, u]+v[c, w, u]+[c, v, u] w \in T^{(k+2)} .
\end{aligned}
$$

Hence,

$$
[c, v][u, w]+[c, w][u, v] \in T^{(k+2)} .
$$

It follows from (11)-(14) that $3[c u, v, w] \in T^{(k+2)}$, as required. The proof of Lemma 2.4 is completed. 
Now we are in a position to prove the theorem. We need to check that

$$
3\left[a_{1}, \ldots, a_{m}\right]\left[b_{1}, \ldots, b_{n}\right] \in T^{(m+n-1)}
$$

if either $m$ or $n$ is odd. Since $\left[\left[a_{1}, \ldots, a_{m}\right],\left[b_{1}, \ldots, b_{n}\right]\right] \in T^{(m+n)} \subseteq T^{(m+n-1)}$, we may assume without loss of generality that $m$ is odd.

The proof is by induction on $m$. If $m=1$ then the theorem clearly holds. Let $m=2 k+1, k>0$. Suppose that

for all $n>0$ and all $a_{i}, b_{j} \in A$.

$$
3\left[a_{1}, \ldots, a_{m-2}\right]\left[b_{1}, \ldots, b_{n}\right] \in T^{(m+n-3)}
$$

Let $c_{1}=\left[a_{1}, a_{2}, \ldots, a_{m-2}\right], c_{2}=\left[b_{1}, b_{2}, \ldots, b_{n}\right]\left(a_{i}, b_{j} \in A\right)$. By (44), we have

$$
\left[c_{1} c_{2}, v, w\right]=\left[c_{1}, v, w\right] c_{2}+\left[c_{1}, v\right]\left[c_{2}, w\right]+\left[c_{1}, w\right]\left[c_{2}, v\right]+c_{1}\left[c_{2}, v, w\right]
$$

so

$$
\left[c_{1}, v, w\right] c_{2}=\left[c_{1} c_{2}, v, w\right]-\left(\left[c_{1}, v\right]\left[c_{2}, w\right]+\left[c_{1}, w\right]\left[c_{2}, v\right]\right)-c_{1}\left[c_{2}, v, w\right]
$$

for all $v, w \in A$.

By the induction hypothesis, $3 c_{1} c_{2} \in T^{(m+n-3)}$ so, by Lemma 2.4,

$$
3\left[c_{1} c_{2}, v, w\right] \in 3\left[T^{(m+n-3)}, A, A\right] \subset T^{(m+n-1)} .
$$

Again, by the induction hypothesis,

$$
3 c_{1}\left[c_{2}, v, w\right] \in T^{(m+n-1)} .
$$

Further,

$$
\left[c_{1}, v\right]\left[c_{2}, w\right]+\left[c_{1}, w\right]\left[c_{2}, v\right]=\left[v, c_{1}\right]\left[w, c_{2}\right]+\left[v, c_{2}\right]\left[w, c_{1}\right]+\left[\left[c_{1}, w\right],\left[c_{2}, v\right]\right]
$$

where $\left[\left[c_{1}, w\right],\left[c_{2}, v\right]\right] \in T^{(m+n)} \subseteq T^{(m+n-1)}$ and, by (4) ,

$$
\left[v, c_{1}\right]\left[w, c_{2}\right]+\left[v, c_{2}\right]\left[w, c_{1}\right]=\left[v w, c_{1}, c_{2}\right]-v\left[w, c_{1}, c_{2}\right]-\left[v, c_{1}, c_{2}\right] w \in T^{(m+n-1)} .
$$

Hence,

$$
\left[c_{1}, v\right]\left[c_{2}, w\right]+\left[c_{1}, w\right]\left[c_{2}, v\right] \in T^{(m+n-1)} .
$$

It follows from (15) - (18) that $3\left[c_{1}, v, w\right] c_{2} \in T^{(m+n-1)}$ for all $v, w \in A$. Theorem 1.1 follows.

\section{ACKNOWLEDGMENT}

The second author was partially supported by CNPq grant 310331/2015-3.

\section{REFERENCES}

[1] N. Abughazalah, P. Etingof, On properties of the lower central series of associative algebras, J. Algebra Appl. 15 (2016), 1650187 (24 pages), arXiv:1508.00943 [math.RA].

[2] A. Bapat, D. Jordan, Lower central series of free algebras in symmetric tensor categories, J. Algebra 373 (2013) 299-311, arXiv:1001.1375 [math.RA].

[3] S. Bhupatiraju, P. Etingof, D. Jordan, W. Kuszmaul and J. Li, Lower central series of a free associative algebra over the integers and finite fields, J. Algebra 372 (2012) 251-274, arXiv:1203.1893 [math.RA].

[4] Cordwell, K., Fei, T., Zhou, K. On lower central series quotients of finitely generated algebras over $\mathbb{Z}$, J. Algebra 423 (2015) 559-572, arXiv:1309.1237 [math.RA].

[5] E.A. da Costa, A. Krasilnikov, Relations in universal Lie nilpotent associative algebras of class 4, Comm. Algebra 46 (2018) 1367-1386, arXiv:1306.4294 [math.RA]/

[6] R.R. Dangovski, On the maximal containments of lower central series ideals, arXiv:1509.08030 [math.RA].

[7] G. Deryabina, A. Krasilnikov, The torsion subgroup of the additive group of a Lie nilpotent associative ring of class 3, J. Algebra 428 (2015) 230-255, arXiv:1308.4172 [math.RA].

[8] G. Deryabina, A. Krasilnikov, Products of commutators in a Lie nilpotent associative algebra, J. Algebra 469 (2017) 84-95, arXiv:1509.08890 [math.RA].

[9] G. Deryabina, A. Krasilnikov, Products of several commutators in a Lie nilpotent associative algebra, Internat. J. Algebra Comput. 27 (2017) 1027-1040, arXiv:1610.03136 [math.RA].

[10] P. Etingof, J. Kim, X. Ma, On universal Lie nilpotent associative algebras, J. Algebra 321 (2009) 697-703, arXiv:0805.1909 [math.RA]. 
[11] A.S. Gordienko, Codimensions of commutators of length 4, Russian Math. Surveys 62 (2007) 187-188.

[12] A.V. Grishin, S.V. Pchelintsev, On the centers of relatively free algebras with an identity of Lie nilpotency, Sb. Math. 206 (2015) 1610-1627.

[13] N. Gupta, F. Levin, On the Lie ideals of a ring, J. Algebra 81 (1983) 225-231.

[14] A. Krasilnikov, The additive group of a Lie nilpotent associative ring, J. Algebra 392 (2013) 10-22, arXiv:1204.2674 [math.RA].

[15] V.N. Latyshev, On the finiteness of the number of generators of a T-ideal with an element $\left[x_{1}, x_{2}, x_{3}, x_{4}\right]$, Sibirsk Mat. Zh. 6 (1965) 1432-1434 (in Russian).

[16] F. Levin, S. Sehgal, On Lie nilpotent group rings, J. Pure Appl. Algebra 37 (1985) 33-39.

[17] I.Z. Monteiro Alves, V. Petrogradsky, Lie structure of truncated symmetric Poisson algebras, J. Algebra 488 (2017) 244-281, arXiv:1612.08051 [math.RA].

[18] R.K. Sharma, J.B. Srivastava, Lie ideals in group rings, J. Pure Appl. Algebra 63 (1990) 67-80.

[19] I.B. Volichenko, The $T$-ideal generated by the element $\left[x_{1}, x_{2}, x_{3}, x_{4}\right]$, Preprint no. 22, Inst. Math. Acad. Sci. Beloruss. SSR (1978) (in Russian).

Department of Computational Mathematics and Mathematical Physics (FS-11), Bauman Moscow State Technical University, 2-nd Baumanskaya Street, 5, 105005 Moscow, Russia

GALINA_DERYABINA@MAIL.RU

Departamento de Matemática, Universidade de Brasília, 70910-900 Brasília, DF, Brasil ALEXEI@UNB.BR 